Nota

\title{
ESCALA FENOLÓGICA DETALHADA DA FASE REPRODUTIVA DE COFFEA ARABICA $\left(^{\mathbf{1}}\right)$
}

\author{
HEVERLY MORAIS $\left({ }^{2,3}\right)$; PAULO HENRIQUE CARAMORI $\left({ }^{2}\right)$; MIRIAN SEI KOGUISHI $\left({ }^{2,3}\right)$; \\ ANA MARIA DE ARRUDA RIBEIRO $\left({ }^{3}\right)$
}

\begin{abstract}
RESUMO
Neste trabalho se propõe uma escala fenológica detalhada para identificar e caracterizar visualmente todas as fases de desenvolvimento reprodutivo do cafeeiro. $\mathrm{O}$ estudo foi realizado em condições de campo com a cultivar IAPAR 59, no período de maio de 2004 a maio de 2005 em Londrina, PR $\left(23^{\circ} 23^{\prime}\right.$ S, 50 ${ }^{\circ} 11^{\prime}$ $\mathrm{W}, 610 \mathrm{~m})$. Periodicamente, foram realizadas medições de crescimento e caracterização evolutiva da morfologia externa das gemas florais, flores e frutos dos ramos plagiotrópicos, em intervalos variados durante todo o ciclo reprodutivo. Na escala proposta, o desenvolvimento reprodutivo do cafeeiro foi dividido em quatro grandes fases: Desenvolvimento da gema floral (G), Floração (FL), Frutificação (F) e Maturação (M). As fases $\mathrm{G}$ e $\mathrm{F}$ foram subdivididas tendo como parâmetro tamanho das gemas e dos frutos, variando de $\mathrm{G}_{1}$ até $\mathrm{G}_{6}$ e $\mathrm{F}_{1}$ até $\mathrm{F}_{6}$. Para descrever a maturação, os frutos foram classificados pela coloração: $\mathrm{M}_{1}$-verde; $\mathrm{M}_{2}$ - verde-cana; $\mathrm{M}_{3}$-vermelho-claro (cereja); $\mathrm{M}_{4}$ - vermelho-escuro (passa) e $\mathrm{M}_{5}$ - preto (seco). A escala proposta pode subsidiar estudos de crescimento, produtividade e manejo da lavoura cafeeira.
\end{abstract}

Palavras-chave: Gema floral, floração, frutificação, estádios de desenvolvimento.

\section{ABSTRACT \\ DETAILED PHENOLOGICAL SCALE OF THE REPRODUCTIVE PHASE OF COFFEA ARABICA}

In this paper is proposed a detailed phenological scale to identify and characterize the reproductive cycle of Coffea arabica. The study was carried out under field conditions using IAPAR 59 cultivar, from May 2004 to May 2005 in Londrina, state of Parana, Brazil (2323' S, 50²11' W, 610 m). Periodically measurements of growth and evolution of the external morphology of the floral buds, flowers and fruits of the plagiotropic branches were taken. In the proposed scale, the reproductive development of coffee was divided in four main phases: Floral bud development $(\mathrm{G})$, Flowering (FL), Fructification (F), and Maturation (M). The phases $G$ and $F$ were subdivided based on bud and fruit size, varying from $G_{1}$ to $G_{6}$ and $F_{1}$ to $F_{6}$. To describe maturation, the fruits were classified according to external color: $M_{1}-$ green, $\mathrm{M}_{2}$ - yellowish, $\mathrm{M}_{3}$ - light red (cherry), $\mathrm{M}_{4}$ - dark red, and $\mathrm{M}_{5}$ - black (dry). The proposed scale could subsidize studies of growth, productivity and management of the coffee crop.

Key words: Flower bud, flowering, fructification, phenological scale.

$\left({ }^{1}\right)$ Recebido para publicação em 12 de fevereiro de 2007 e aceito em 20 de agosto de 2007.

$\left({ }^{2}\right)$ Área de Ecofisiologia, IAPAR, Caixa Postal 481, 86001-970 Londrina (PR). E-mail: heverly@iapar.br, caramori@iapar.br $\left(^{*}\right)$ Autor correspondente; miriansei@iapar.br.

$\left({ }^{3}\right)$ Universidade Estadual de Londrina, Centro de Ciências Agrárias, Departamento de Agronomia, Caixa Postal 6001, 86051-990 Londrina (PR), Brasil. E-mail: arruda@uel.br. 


\section{Introdução}

A descrição das etapas de crescimento e desenvolvimento das plantas incluindo monocotiledôneas, dicotiledôneas, gramíneas e perenes, sempre foi uma questão de interesse da pesquisa. O calendário fenológico existe na China há mais de dois mil anos. O período em que a cerejeira inicia a floração foi registrado em Kyoto no ano de 750 d.C., e desde 1736 os dados fenológicos têm sido documentados por sucessivas gerações (LARCHER, 2000). Nos dias atuais, o conhecimento da fenologia auxilia no planejamento das épocas oportunas para a realização de práticas culturais como aplicação de fertilizantes, controle de pragas, doenças e ervas daninhas (ARCILA et al., 1998), bem como em pesquisas de estimativas de safra, previsão da época de maturação e programas de melhoramento (PEZzOPANE et al., 2003).

A fase reprodutiva é marcada pela capacidade da planta em produzir flores e resultante de mudanças que ocorrem no meristema das gemas (LARcher, 2000). No cafeeiro, o desenvolvimento reprodutivo começa com a iniciação floral e termina com a queda dos frutos. CAMARGO e CAMARGo (2001) subdividiram o ciclo fenológico completo do cafeeiro em seis fases: 1) vegetação e gemas foliares; 2) indução e maturação das gemas florais; 3) florada; 4) granação dos frutos; 5) maturação dos frutos; 6) repouso e senescência dos ramos terciários e quaternários. Pezzopane et al. (2003) descreveram uma escala das fases fenológicas reprodutivas do cafeeiro com base em números: 0 (gema dormente); 1 (gema intumescida); 2 (abotoado); 3 (florada); 4 (pós-florada); 5 (chumbinho); 6 (expansão dos frutos); 7 (grão verde); 8 (verde-cana); 9 (cereja); 10 (passa); 11 (seco). A análise histológica é o melhor método para avaliar com precisão a transição das fenofases (MoENs, 1963; BARros et al., 1978), mas é de difícil utilização prática.

Este trabalho tem como objetivo elaborar uma escala detalhada da fase reprodutiva do cafeeiro, fornecendo suporte para a caracterização do desenvolvimento reprodutivo da espécie Coffea arabica.

O experimento foi desenvolvido na área experimental do IAPAR, em Londrina (PR), cujas coordenadas geográficas são: altitude $610 \mathrm{~m}$, latitude $23^{\circ} 23^{\prime} \mathrm{S}$ e longitude $50^{\circ} 11^{\prime} \mathrm{W}$. O solo é do tipo Latossolo Vermelho Eutroférrico (EMBRAPA, 1999), com $82 \%$ de argila. O clima da região é do tipo Cfa, descrito como clima subtropical úmido com verão quente, segundo a classificação de Köppen. A temperatura média anual é $21^{\circ} \mathrm{C}$, a média do mês mais quente é $24^{\circ} \mathrm{C}$ (janeiro) e a média do mês mais frio é $17^{\circ} \mathrm{C}$ (junho). A precipitação média anual é de 1.500 $\mathrm{mm}$, sendo dezembro, janeiro e fevereiro os meses mais chuvosos e junho, julho e agosto os mais secos (CAVIGLIONE et al., 2000).

Foram avaliados cafeeiros da espécie Coffea arabica da cultivar IAPAR 59, plantados em junho de 1993 no espaçamento de 2,5 m entre linhas e 1,5 m entre plantas com 2 plantas por cova, em uma área total de $1600 \mathrm{~m}^{2}$ (20 m x $\left.80 \mathrm{~m}\right)$. Essas plantas foram recepadas em outubro de 2000, em decorrência de geada. Foram avaliadas quatro parcelas de quatro plantas de cafeeiros cultivados a pleno sol. Para tanto foram marcados e avaliados dois ramos localizados no terço superior de cada planta: um voltado para a direção norte e outro para a direção sul. Nos ramos marcados, foram avaliados oito nós produtivos crescidos no ano anterior. As observações dos estádios de desenvolvimento foram efetuadas por meio de visualização externa do tamanho das gemas florais, quantidades de flores e coloração dos frutos. As avaliações foram realizadas de maio de 2004 a maio de 2005, nas seguintes datas: 8/5/04, 27/5/04, 22/ 6/04 21/7/04 20/8/04, 15/9/04 20/9/04, 27/9/04, 20/10/04, 9/11/04, 24/11/04, 15/12/04, 27/1/05, $15 / 3 / 2005,29 / 3 / 2005,12 / 4 / 2005,26 / 4 / 2005,10 /$ $5 / 2005$ e 20/5/2005. Observações semanais foram feitas a fim de detectar as modificações e/ou desenvolvimento das estruturas avaliadas.

O desenvolvimento reprodutivo do cafeeiro (Figura 1) foi dividido em quatro grandes fases: desenvolvimento da gema floral (G), floração (FL), frutificação (F) e maturação (M), as quais são denominadas com suas letras iniciais, a exemplo de método adotado por MARUR e RUANO (2001) para a determinação dos estádios de desenvolvimento do algodoeiro. A fase $G$ foi subdividida tendo como variável o tamanho das gemas; a fase $\mathrm{F}$ foi subdividida de acordo com o tamanho dos frutos e para a fase $M$ o critério adotado foi coloração dos frutos. Cada fase do desenvolvimento é acompanhada por alterações morfológicas, anatômicas e bioquímicas, sendo sua duração variável e decorrente de fatores ambientais (chuva, temperatura e fotoperíodo) e atributos endógenos (genótipo, crescimento vegetativo, potencial produtivo estado nutricional e reguladores de crescimento).

A passagem da gema à fase reprodutiva (indução) compreende uma seqüência de eventos de natureza bioquímica e morfológica que começa com o estímulo indutivo. Em Coffea arabica um leve achatamento do meristema e sua elevação acima do nível original são os primeiros indícios visuais de transição floral (RENA e MAESTRI, 1985). Na seqüência são lançadas pequenas gemas que vão crescendo e ficando visivelmente diferenciadas. 


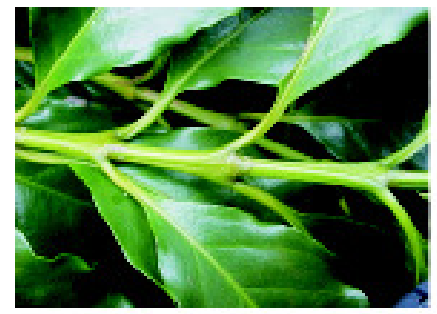

$\mathrm{G}_{1}$

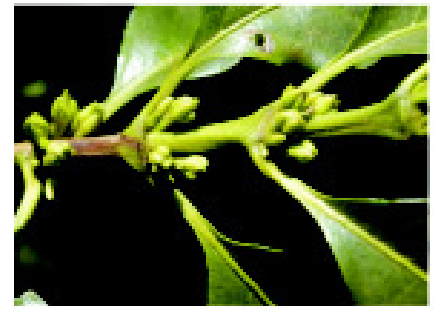

$\mathrm{G}_{4}$

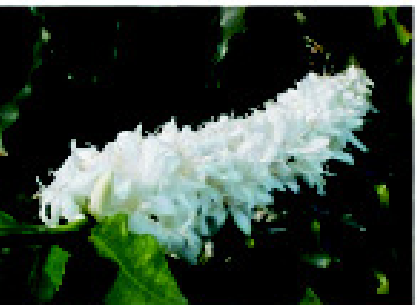

FL

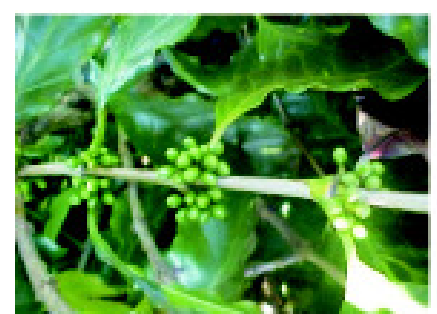

$\mathrm{F}_{3}$

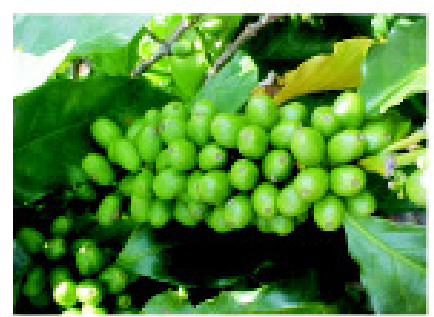

$\mathrm{F}_{6}$

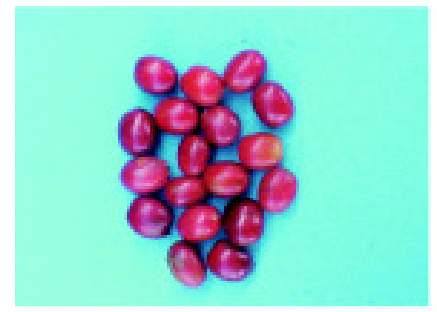

$\mathrm{M}_{3}$ - Cereja

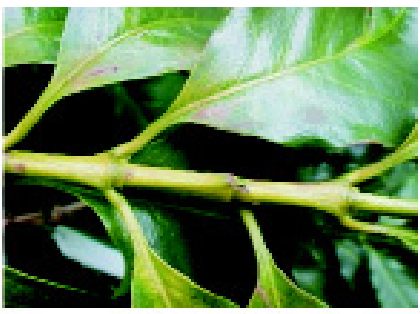

$\mathrm{G}_{2}$

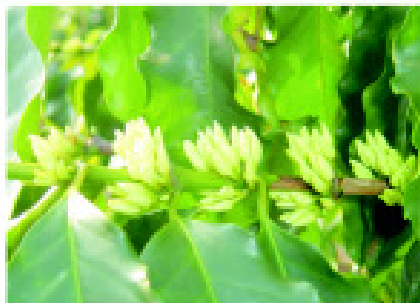

$\mathrm{G}_{3}$

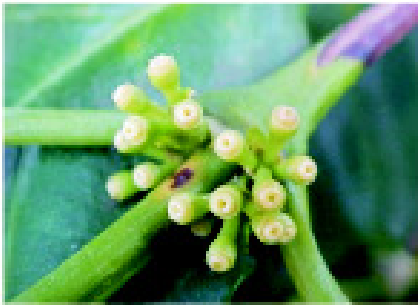

$\mathrm{F}_{1}$

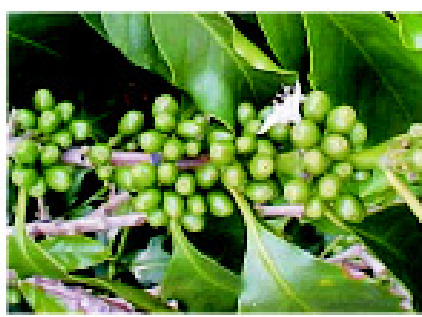

$\mathrm{F}_{4}$

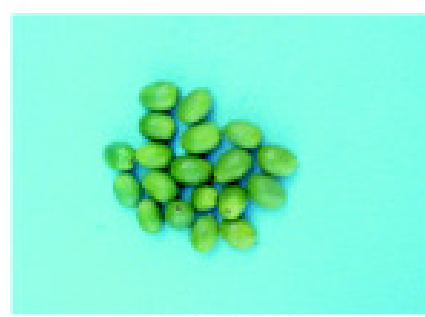

$\mathrm{M}_{1}$ - Verde

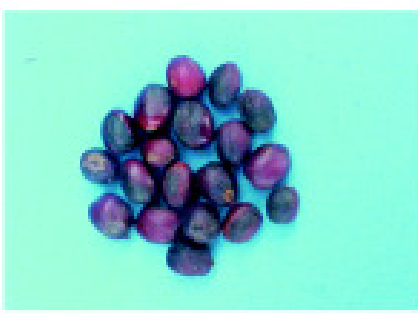

$\mathrm{M}_{4}$ - Passa

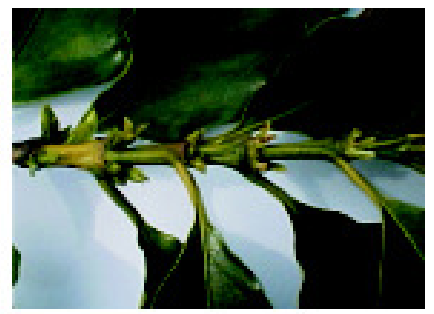

$\mathrm{G}_{3}$

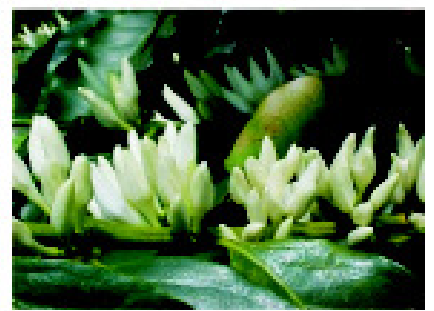

$\mathrm{G}_{6}$

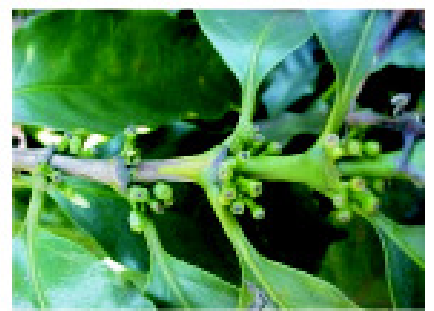

$\mathrm{F}_{2}$

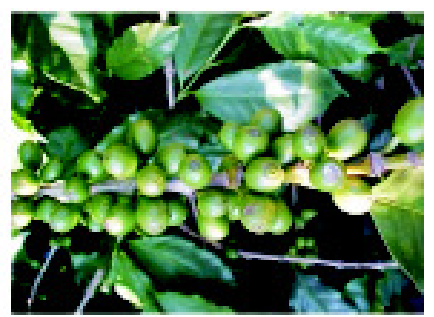

$\mathrm{F}_{5}$

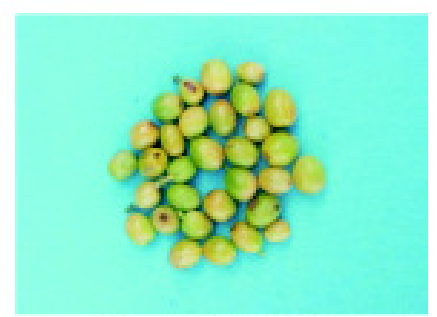

$\mathrm{M}_{2}$ - Verde-cana

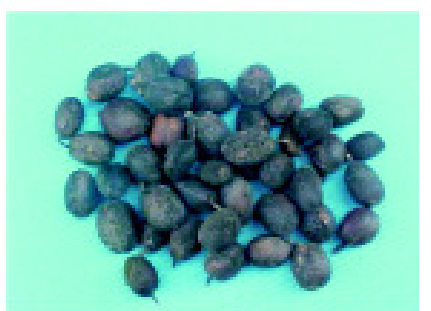

$\mathrm{M}_{5}$ - Seco

Figura 1. Escala das Fases Fenológicas Reprodutivas do Cafeeiro. 
A caracterização das subfases foi feita da seguinte maneira: $G_{1}$ - refere-se aos nós com gemas indiferenciadas; $G_{2}$ - nós com gemas intumescidas; $G_{3}$ gemas com até $3 \mathrm{~mm}$ de comprimento; $\mathrm{G}_{4}$ - gemas medindo 3,1 a 6 mm de comprimento; $\mathrm{G}_{5}$ - gemas de 6,1 a $10 \mathrm{~mm}$ (coloração verde claro); $\mathrm{G}_{6}$ - gema maior que 10 $\mathrm{mm}$ (coloração branca). Após o $\mathrm{G}_{6}$, normalmente ocorre a abertura das flores nas primeiras horas da manhã, começam a murchar no segundo dia e caem no terceiro.

O crescimento e o desenvolvimento dos frutos seguem o padrão de uma curva sigmoidal dupla (Wormer e NuUnguna, 1966; Cannell, 1971; Gómez, 1977). As subfases foram descritas de acordo com o tamanho dos frutos: $\mathrm{F}_{1}$ - até $3 \mathrm{~mm}$ de comprimento; $\mathrm{F}_{2}-3,1$ a $4 \mathrm{~mm} ; \mathrm{F}_{3}-4,1$ a $5 \mathrm{~mm}$; $\mathrm{F}_{4}-5,1$ a $10 \mathrm{~mm} ; \mathrm{F}_{5}-10$ a $15 \mathrm{~mm} ; \mathrm{F}_{6}$ - maior que $15 \mathrm{~mm}$ (Figura 1).

$\mathrm{Na}$ fase de maturação $(\mathrm{M})$, ocorrem vários processos metabólicos e modificações na composição química, que permitem os frutos alcançarem seu ponto ideal de colheita, o qual se confirma por troca de coloração de verde a vermelho ou amarelo, dependendo da cultivar. Neste trabalho, a descrição das subfases da maturação iniciou quando foram identificados nas observações semanais frutos de coloração diferente do verde. Assim designou-se $M_{1}$ para frutos de coloração verde, ou seja, sem evidências de alteração na cor; $M_{2}$ para frutos de coloração verde-cana, os quais já iniciaram a maturação; $\mathrm{M}_{3}$ para frutos em estádio "cereja", de coloração vermelhoclaro e maduros fisiologicamente; $\mathrm{M}_{4}$ para frutos no estádio "passa", de coloração vermelho-escuro e com início de desidratação; $\mathrm{M}_{5}$ para frutos secos, desidratados com coloração externa escura (Figura 1). Outros autores também descreveram os estádios de desenvolvimento reprodutivo do cafeeiro (Moens, 1968; Gouveia, 1984; Camoyo e Arcila, 1996; Pezzopane et al., 2003).

A descrição das etapas de desenvolvimento reprodutivo do cafeeiro, conforme proposta nessa escala detalhada, utilizando critérios de fácil identificação visual, auxilia no desenvolvimento de modelos de crescimento e desenvolvimento do cafeeiro; na definição e aperfeiçoamento de práticas de manejo, como adubação e controle de pragas; e na identificação de eventuais distúrbios que possam ocorrer em cada fase proposta, com a adoção de medidas corretivas sempre que possível.

\section{REFERÊNCIAS}

ARCILA, P.J.; BUHR, L.; BLEIHOLDER, H.; HACK, H.; WICKE, H. Aplicación de la Escala BBCH ampliada para la descripción de las fases fenologicas del desarrollo de la planta de café Coffea sp. Chinchina: Cenicafé, 1998. (Folheto interno)
BARROS, R.S.; MAESTRI, M.; COONS, M.P. The physiology of flowering in coffee: a review. Journal of Coffee Research, Balehonnur, v.8, n.2-3, p.29-73, 1978.

CAMARGO, A.P.; CAMARGO, M.B.P. Definição e esquematização das fases fenológicas do cafeeiro arábica nas condições tropicais do Brasil. Bragantia, Campinas, v.20, n.1, p.65-68, 2001.

CAMOYO V.G.C.; ARCILA P.J. Estudio anatómico y morfológico de la diferenciación y desarrollo de las flores del cafeto Coffea arabica L. variedad Colombia. Cenicafé, Chinchina, v.47, n.3, p.121-139, 1996.

CANNELL, M.G.R. Effect of the presence of fruits on net photosynthesis. Annual Report Coffee Research Station, Ruiru, 1971. p.41-42.

CAVIGLIONE, J.H.; CARAMORI, P.H.; KIIHL, L.B; OLIVEIRA, D. Cartas climáticas do Paraná. Londrina, Brasil: IAPAR, 2000. CD-ROM.

EMPRESA BRASILEIRA DE PESQUISA AGROPECUÁRIA. Sistema brasileiro de classificação de solos. Rio de Janeiro: EMBRAPA-CNPS, 1999.

GÓMEZ G.L. Influencia de los factores climáticos sobre la periodicidad de crecimiento del cafeto. Cenicafé, Chinchina, v.28, n.1, p.3-17, 1977.

GOUVEIA, N.M. Estudo da diferenciação e crescimento das gemas florais de Coffea arabica L.: observações sobre a antese e maturação dos frutos. 1984. Dissertação (Mestrado em Ciências) - Universidade Estadual de Campinas, Campinas.

LARCHER, W. Ecofisiologia vegetal. São Carlos: RiMa, 2000.

MARUR, C.J.; RUANO, O. A reference system for determination of developmental stages of upland cotton. Revista de Oleaginosas e Fibrosas, Campina Grande, v.5, n.2, p.313-317, 2001.

MES, M.G. Studies on the flowering of Coffea arabica L. III Various phenomena associated with the dormancy of coffee flower buds. Portugaliae Acta Biologica, Lisboa, n.5, p.25-44, 1957.

MOENS, P. Investigaciones morfológicas, ecológicas y fisiológicas sobre cafetos. Turrialba, San Jose, v.18, n.3, p.209$233,1968$.

MOENS, P. Les bougeons végétatifs et génératifs de Coffea canephora Pierre. La Cellule, Louvain, v.63, n.2, p.165-244, 1963.

PEZZOPANE, J.R.M.; PEDRO JÚNIOR, M.J.; THOMAZIELLO, R.A.; CAMARGO, M.B.P. Escala para avaliação de estádios fenológicos do cafeeiro arábica. Bragantia, Campinas, v.62, n.3, p.499-505, 2003.

RENA, A.B.; MAESTRI, M. Fisiologia do cafeeiro. Informe Agropecuário, Belo Horizonte, v.11, p.26-40, 1985.

WORMER, T.M.; NJUNGUNA, S.G. Bean size and shape as quality factors in Kenya Coffee. Kenya Coffee, Nairobi, n.31, p.397-405, 1966. 